\title{
In vitro antiviral activity of ribavirin against severe fever with thrombocytopenia syndrome virus
}

\author{
Myung Jin Lee ${ }^{1, *}$, Kye-Hyung Kim ${ }^{1, \dagger}$, Jongyoun $\mathrm{Yi}^{2}$, Su Jin Choi ${ }^{3}$, Pyoeng Gyun Choe ${ }^{1}$, Wan Beom Park ${ }^{1}$, \\ Nam Joong Kim ${ }^{1}$, and Myoung-don $\mathrm{Oh}^{1}$
}

\begin{abstract}
${ }^{1}$ Department of Internal Medicine, Seoul National University College of Medicine, Seoul; ${ }^{2}$ Department of Laboratory Medicine, Pusan National University School of Medicine, Busan; ${ }^{3}$ Laboratory of Infection \& Immunity, Seoul National University Hospital Biomedical Research

Institute, Seoul, Korea
\end{abstract}

Received: April 1, 2016

Revised : June 20, 2016

Accepted: July 8, 2016

\section{Correspondence to}

Myoung-don Oh, M.D.

Department of Internal

Medicine, Seoul National

University College of Medicine, 101 Daehak-ro, Jongno-gu, Seoul 03080, Korea

Tel: +82-2-2072-2945

Fax: +82-2-762-9662

E-mail:mdohmd@snu.ac.kr

* Current affiliation: Department of Internal Medicine, Gachon University Gil Medical Center, Incheon, Korea

${ }^{\dagger}$ Current affiliation: Department of Internal Medicine, Pusan

National University School of

Medicine, Busan, Korea
Background/Aims: Severe fever with thrombocytopenia syndrome (SFTS) is an emerging infectious disease caused by severe fever with thrombocytopenia syndrome virus (SFTSV), a novel bunyavirus. As yet, there is no effective antiviral therapy for SFTS. Ribavirin is a broad-spectrum antiviral agent, which has been tried for treatment of SFTS. In this study, antiviral activity of ribavirin against SFTSV has been investigated.

Methods: Vero cell-grown SFTSV strain Gangwon/Korea/2012 was treated with ribavirin at various concentrations. Antiviral activity of ribavirin was evaluated by inhibition of the SFTSV cytopathic effect in Vero cells and quantification of viral RNA load in culture supernatant using one-step real-time reverse transcription polymerase chain reaction. Cytotoxicity of ribavirin was determined by a tetrazolium-based colorimetric method.

Results: Ribavirin reduced SFTSV titers in a dose-dependent manner, with a half-maximal inhibitory concentration ranged from 3.69 to $8.72 \mu \mathrm{g} / \mathrm{mL}$. Cytopathic effects were reduced as ribavirin concentration increased. No significant cytotoxicity was detected at ribavirin concentrations of $\leq 31.3 \mu \mathrm{g} / \mathrm{mL}$.

Conclusions: Ribavirin exhibited inhibitory activity against SFTSV replication in vitro, which suggests that ribavirin can be used as a potential antiviral agent for SFTS.

Keywords: Bunyaviridae infections; Phlebovirus; Ribavirin; Antiviral agents

\section{INTRODUCTION}

Severe fever with thrombocytopenia syndrome (SFTS) is an emerging tick-borne viral disease. It was first observed in central China in 2009 and was subsequently reported from Japan and Korea [1-3]. The etiological agent of SFTS is a novel phlebovirus called severe fever with thrombocytopenia syndrome virus (SFTSV), which belongs to the family Bunyaviridae [1]. The major clinical features of SFTS include fever, vomiting, diarrhea, thrombocytopenia, leukopenia, and multi-organ dysfunction $[1-3]$. The case fatality rate of SFTS is between $6 \%$ and $30 \%$; however, there are neither antiviral agents nor vaccines currently proven to be effective against 
SFTSV [1-4].

Ribavirin (1- $\beta$-D-ribofuranosyl-1,2,4-triazole-3-carboxyadmide) is a synthetic nucleoside analogue with antiviral activity against a variety of DNA and RNA viruses [5]. Because ribavirin exhibits antiviral effects against other bunyavirus infections, including Crimean-Congo hemorrhagic fever (CCHF), Rift Valley fever, and hemorrhagic fever with renal syndrome $[5,6]$, it has been anecdotally used in the treatment of SFTS $[4,7]$. However, its efficacy against SFTSV has yet to be evaluated directly, as previous study by Liu et al. [4] was a clinical observational study. Therefore, we aimed to assess the antiviral activity of ribavirin in an in vitro model of SFTSV replication in this study.

\section{METHODS}

\section{Cell lines, viruses, and compounds}

The Vero cells (KCLB no.10081) purchased from the Korean Cell Line Bank (KCLB, Seoul, Korea) were maintained and propagated in RPMI 1640 medium(Welgene, Daegu, Korea) supplemented with $2 \%$ heat-inactivated fetal bovine serum (Welgene) and $1 \%$ penicillin-streptomycin (Gibco, Carlsbad, CA, USA). The cells were incubated at $37^{\circ} \mathrm{C}$ in a humidified, $5 \% \mathrm{CO}_{2}$ chamber. Viral stocks derived from SFTSV strain Gangwon/Korea/2012 [3] were used and the 50\% tissue culture infective dose $\left(\mathrm{TCID}_{50}\right)$ was calculated as described by Reed and Muench [8]. Ribavirin (Sigma-Aldrich, St. Louis, MO, USA) in the form of a powder was dissolved in phosphate-buffered saline (PBS) to obtain a stock solution of $10 \mathrm{mg} / \mathrm{mL}$. Working concentrations of the drug was prepared immediately before use from the stock solution diluted in RPMI medium.

\section{Cell viability assay}

TheMTS (3-[4,5-dimethylthiazol-2-yl]-5-[3-carboxymethoxyphenyl]-2-[4-sulfophenyl]-2H-tetrazolium) assay (Promega, Madison, WI, USA) was performed to determine cellular toxicity of ribavirin. Briefly, Vero cells $\left(1 \times 10^{5}\right.$ cells $\left./ \mathrm{mL}\right)$ seeded in 96-well tissue culture plates were incubated overnight. Increasing concentrations of ribavirin (o to 1,000 $\mu \mathrm{g}$ / $\mathrm{mL}$, in serial 2-fold dilutions) in $100 \mu \mathrm{L}$ of RPMI were added to each well in quadruplicate. At 24, 48, and 72 hours of incubation, the MTS reagent was added to each well and tissue culture plates were incubated for another 4 hours. Thereafter, their optical densities were measured at 490 nm using a Versamax microplate reader (Molecular Devices, Sunnyvale, CA, USA). The ribavirin concentrations were considered cytotoxic if their optical densities were significantly reduced compared to those of mock-treated controls with $p<0.05$ by one-way analysis of variance (ANOVA).

\section{Antiviral assay}

Confluent monolayers of Vero cells $\left(1 \times 10^{5}\right.$ cells $\left./ \mathrm{mL}\right)$ in 96-well tissue culture plates were infected with 100x the TCID $_{50}$ of SFTSV. After 1 hour of viral adsorption, the inoculum was removed and the cells were washed three times with PBS to remove unbound virus. The cells were then treated in quadruplicate with ribavirin concentrations ranging from o to $32 \mu \mathrm{g} / \mathrm{mL}$ prepared in $100 \mu \mathrm{L}$ of culture medium. At 12, 24, and 48 hours posttreatment (hpt), the cytopathic effects (CPE) were observed under light microscopy and culture supernatants from the replicates were pooled together to quantify SFTSV RNA load.

SFTSV viral titers in culture supernatants were measured by a one-step real-time reverse transcription polymerase chain reaction (RT-PCR) with amplification of the partial large (L) segment of SFTSV. RNA was extracted from the supernatants of each ribavirin-treated sample using the QIAamp viral RNA kit (Qiagen, Hilden, Germany), as described by the manufacturer. We used the GoTaq Probe one-step RT-qPCR system (Promega) and the PCR primers and probes of $L$ segment were modified from those used in a previous study [9]: forward primer 5'-AGTCTAGGTCATCTGATCCGTTYAG-3'; reverse primer 5'-TGTAAG TTCGYCCTTTGTCCAT-3'; probe 5'-CAATGACAGAYGCCTTCCATGGYAATAGGG- 3 '. Real-time PCR cycling was performed on an ABI 7500 (Applied Biosystems, Foster City, CA, USA) as follows: reverse transcription at $45^{\circ} \mathrm{C}$ for 15 minutes, 1 cycle at $95^{\circ} \mathrm{C}$ for 10 minutes, and 40 cycles at $95^{\circ} \mathrm{C}$ for 15 seconds and $60^{\circ} \mathrm{C}$ for 60 seconds. The cut-off threshold cycle (Ct) value for a positive sample was set at 35 cycles. Serial 10-fold dilutions from $10^{4}$ to $10^{-2} \mathrm{TCID}_{50} / \mathrm{mL}$ of SFTSV transcript RNAs were used to generate a standard curve of RT-PCR. The Ct values of viral RNA titers in the samples were obtained and viral loads were calculated by transforming the Ct values in terms of the standard curve us- 

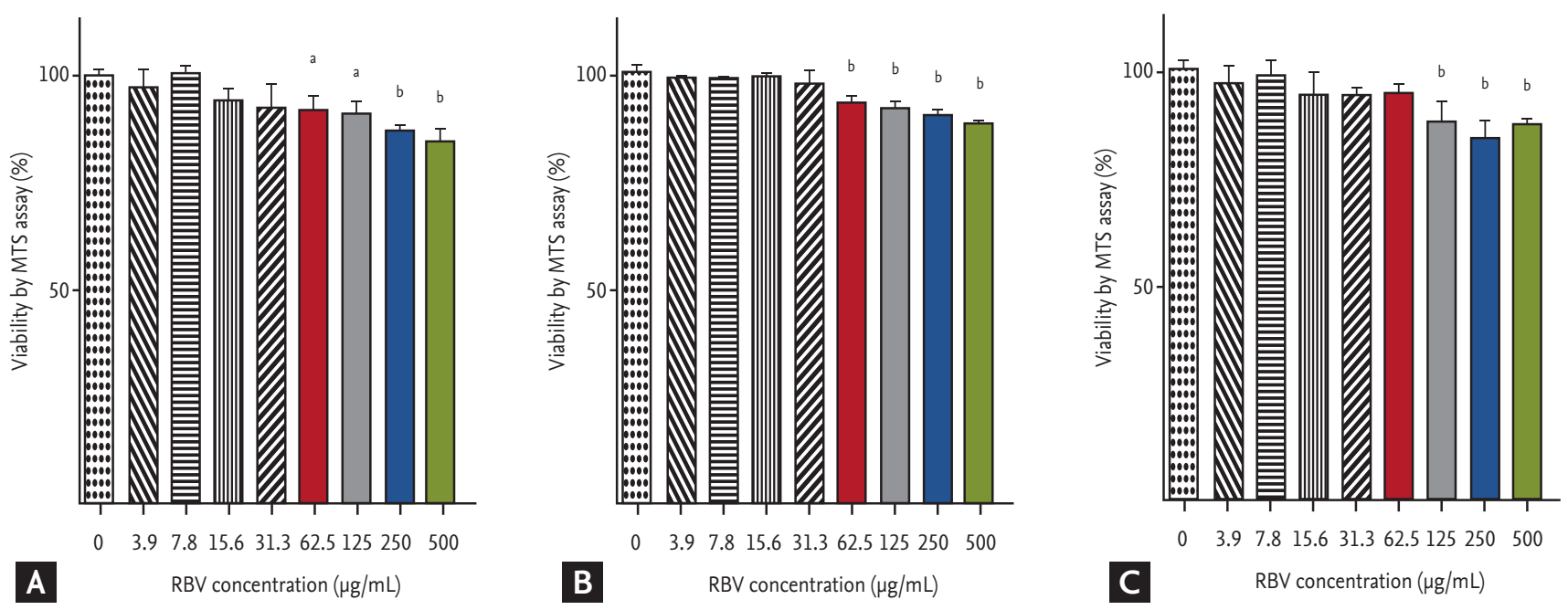

Figure 1. Effect of ribavirin (RBV) on cell viability of Vero cells. MTS (3-[4,5-dimethylthiazol-2-yl]-5-[3-carboxymethoxyphenyl]-2-[4-sulfophenyl]-2H-tetrazolium) assay was done at 24,48 , and 72 hours posttreatment (hpt) for uninfected Vero cells, as described in METHODS. (A) $24 \mathrm{hpt,} \mathrm{(B)} 48 \mathrm{hpt}$, and (C) $72 \mathrm{hpt}$. The data represent the mean \pm standard error of mean of four replicate wells and are expressed as the percentage of untreated controls. One-way analysis of variance with Dunnet's multiple comparison test (GraphPad Prism 5, GraphPad Software) was used for the data analysis. ${ }^{a} p<0.05,{ }^{b} p<0.001$ as compared to RBV-untreated Vero cells.

ing the ABI 7500 software version 2.0.5 (Applied Biosystems). The viral titers are expressed as $\log _{10} \mathrm{TCID}_{50} / \mathrm{mL}$ \pm standard error of mean.

All experiments with live SFTSV were performed in a biosafety level 3 laboratory at Seoul National University Biomedical Research Institute.

\section{Data analysis}

Data obtained from antiviral assay were analyzed by fitting a curve using non-linear regression with the goodness of fit tested by $r^{2}$. The half-maximal inhibitory concentration $\left(\mathrm{IC}_{50}\right)$ of ribavirin was calculated from a sigmoidal dose-response curve. Statistical differences between the viral titers of each sample at different time point were examined using a one-way ANOVA followed by Dunnet's multiple comparison tests. $p$ values of $<0.05$ were considered to be statistically significant. All analyses were performed using GraphPad Prism version 5 (GraphPad Software, San Diego, CA, USA).

\section{RESULTS}

\section{Effects of ribavirin on cell viability}

No significant decrease in cell viability in ribavirin-treated Vero cells was detected for ribavirin at concentrations $\leq 31.3 \mu \mathrm{g} / \mathrm{mL}$ at 24,48 , and $72 \mathrm{hpt}$ (Fig. 1). By contrast, cell viability was decreased at ribavirin concentrations $\geq$ $62.5 \mu \mathrm{g} / \mathrm{mL}$ at 12 and $24 \mathrm{hpt}(p<0.05$ and $p<0.001)$, and at concentrations $\geq 125 \mu \mathrm{g} / \mathrm{mL}$ at $72 \mathrm{hpt}$ of ribavirin $(p$ $<0.001$ ). Based on these results, we performed the antiviral assays at non-cytotoxic ribavirin concentrations ranging from o to $32 \mu \mathrm{g} / \mathrm{mL}$.

\section{Antiviral activity}

Vero cells were monitored for CPEs at 12, 24, and $48 \mathrm{hpt}$ of ribavirin. While there were no obvious CPEs at $12 \mathrm{hpt}$, a dose-dependent reduction in CPEs in ribavirin-treated cells was observed at $24 \mathrm{hpt}$. A clear inhibition of CPEs was identified at ribavirin concentrations $\geq 16 \mu \mathrm{g} /$ $\mathrm{mL}$ at 48 hpt (Fig. 2).

Ribavirin also showed a dose-dependent inhibitory effect on viral RNA replication (Fig. 3). Viral titers were significantly reduced at $12 \mathrm{hpt}(p<0.001)$ in cells treated with $\geq 8 \mu \mathrm{g}$ ribavirin $/ \mathrm{mL}$. At $24 \mathrm{hpt}$, there was a dose-dependent reduction in viral RNA titers $\left(r^{2}>0.9\right)$ in cells treated with ribavirin concentrations from 1 to $32 \mu \mathrm{g} / \mathrm{mL}$ $(p<0.01)$. The inhibitory effect of ribavirin concentrations $\geq 16 \mu \mathrm{g} / \mathrm{mL}$ persisted for 48 hours. The $\mathrm{IC}_{50}$ range of ribavirin in our in vitro model of SFTSV infection was 3.69 to $8.72 \mu \mathrm{g} / \mathrm{mL}$, as determined from the viral titers of culture supernatants collected at 24 and 48 hpt (Fig. 4). 


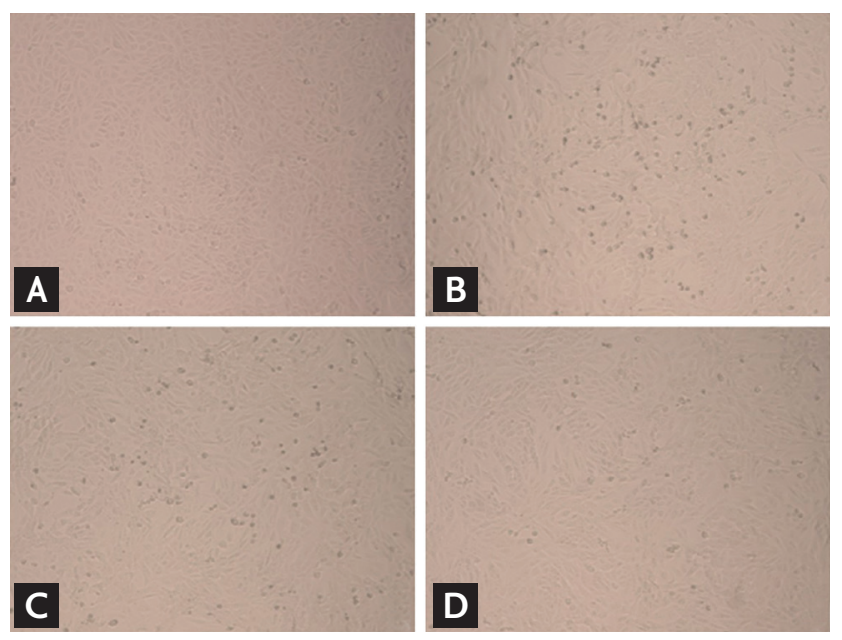

Figure 2. Inhibition of cytopathic effect (CPE) of severe fever with thrombocytopenia syndrome virus (SFTSV)-infected Vero cells by ribavirin. The CPE of infected cells was observed microscopically 48 hours posttreatment (hpt). Treatment of ribavirin at concentrations $\geq 16 \mu \mathrm{g} / \mathrm{mL}$ significantly reduced CPE formations. (A) Uninfected Vero cell controls, (B) SFTSV-infected Vero cells without ribavirin treatment, (C) SFTSV-infected Vero cells treated with $4 \mu \mathrm{g} / \mathrm{mL}$ of ribavirin, (D) SFTSV-infected Vero cells treated with $16 \mu \mathrm{g} / \mathrm{mL}$ of ribavirin. All images were captured at 100x magnification.

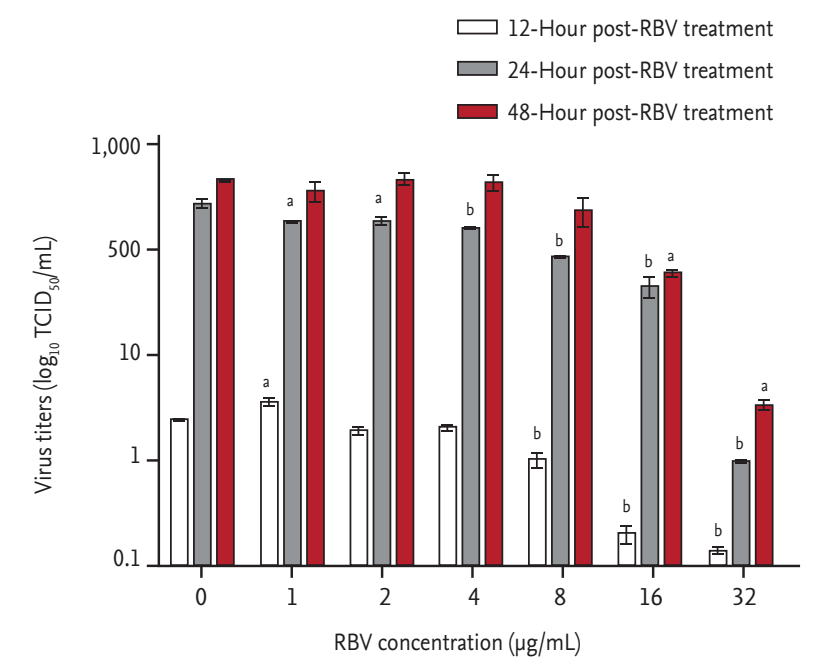

Figure 3. In vitro inhibitory effect of ribavirin (RBV) against severe fever with thrombocytopenia syndrome virus (SFTSV) replication in Vero cells. After their infection with 100x $50 \%$ tissue culture infective dose $\left(\right.$ TCID $\left._{50}\right)$ of SFTSV for 1 hour, Vero cells were treated with increasing concentrations of RBV. Culture supernatants were harvested at the indicated times and their viral RNA titers were assayed using real-time reverse transcription polymerase chain reaction. A one-way analysis of variance with Dunnet's multiple comparison test (GraphPad Prism 5, GraphPad Software) was used for the data analysis. The data are mean \pm standard error of mean. ${ }^{a} p<0.01,{ }^{b} p<0.001$ as compared to mock-treated cells (indicated as o $\mu \mathrm{g} \mathrm{RBV} / \mathrm{mL}$ ).

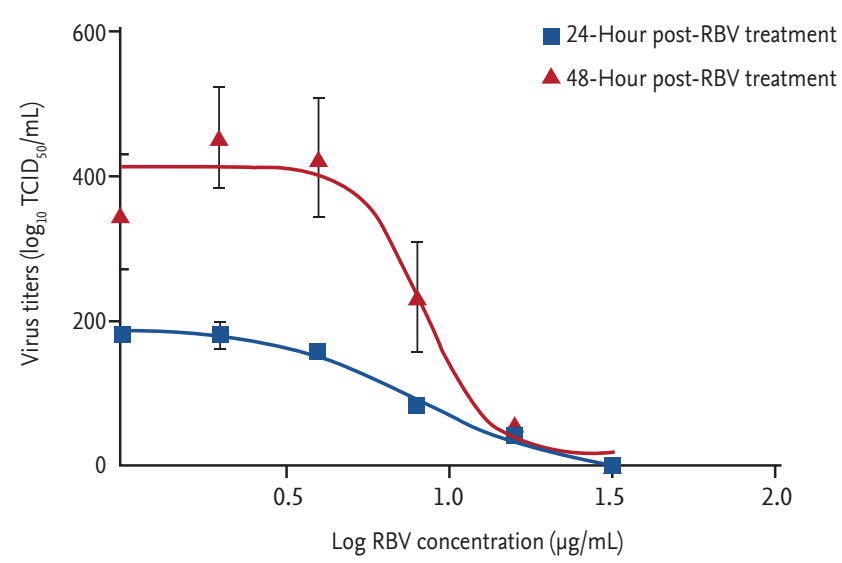

Figure 4. Non-linear regression analysis of the concentration-dependent curves showing the antiviral activity of ribavirin (RBV) against severe fever with thrombocytopenia syndrome virus replication. The half-maximal inhibitory concentration was calculated using GraphPad Prism 5 (GraphPad Software) and fitted using a sigmoidal dose-response algorithm. Data are expressed as mean \pm standard error of mean. TCID $_{50}, 50 \%$ tissue culture infective dose.

\section{DISCUSSION}

In this study, we demonstrate that ribavirin inhibits the viral replication of SFTSV in Vero cells in a dose-dependent manner. The $\mathrm{IC}_{50}$ of ribavirin against SFTSV ranged from 3.69 to $8.72 \mu \mathrm{g} / \mathrm{mL}$, which is similar to that for Andes virus (5 to $12.5 \mu \mathrm{g} / \mathrm{mL}$ ) and Lassa virus (9 to 20 $\mu \mathrm{g} / \mathrm{mL}$ ), but substantially lower than that for Rift Valley fever virus (40 to $80 \mu \mathrm{g} / \mathrm{mL}$ ) [5,6]. Our results suggest that ribavirin may be used as an antiviral agent for patients with SFTSV infection.

Our study clearly showed that treatment with ribavirin concentrations of $\geq 8 \mu \mathrm{g} / \mathrm{mL}$ significantly reduced viral RNA titers in vitro. A recent study by Shimojima at al. [10] also reported that SFTS viral production in Vero, Huh7, and $\mathrm{U}_{2} \mathrm{OS}$ cells was reduced by $99 \%$ at ribavirin concentrations of 64,20 , and $19 \mu \mathrm{g} / \mathrm{mL}$, respectively. The actual concentrations of ribavirin showing antiviral effects in other cell lines might be lower than that of Vero cells, which are known to be more resistant to ribavirin [11].

The present study differed from the study by Shimojima et al. [10] in that: (1) we demonstrated antiviral effect of ribavirin in cells treated after viral infection, whereas Shimojima et al. [10] reported inhibitory effect of ribavirin in cells treated before viral infection, not in 
pre-infected cells; (2) we used a Korean SFTSV strain, while they used a Chinese SFTSV strain. Considering the discrepant antiviral activity of ribavirin according to the time point of treatment reported by Shimojima et al. [10], we suspect that there might be a direct action of ribavirin on unbound SFTSV itself, not an inhibition of viral proliferation in cells. However, our results represent that ribavirin inhibited actual viral proliferation in cells as ribavirin was administered after the PBS washing of virus-infected cells. Although we cannot directly compare the infection status of cells before ribavirin treatment in our study with those of Shimojima et al. [10] as different strains of SFTSV were used, a plausible explanation for the different results is that ribavirin may have restricted antiviral activity to the early infected cells, not to fully-infected cells. Similar results have been reported in previous studies with other viruses, which support our assumption on variable efficacy of ribavirin affected by treatment time [12-16]. Ribavirin added on cells at day -1 or o postinfection with CCHF virus were shown to be active, whereas antiviral activity were decreased with addition of ribavirin at day 1 postinfection, and completely lost when ribavirin were added at day 2 and 3 postinfection [12]. Clinically, CCHF patients with early ribavirin use had a lower case fatality rate than those with late ribavirin use, and were less likely to progress to severe diseases $[13,14]$. Similarly, antiviral effect of ribavirin in vesicular stomatitis virus-infected cells was reduced as the time of addition was delayed [15]. In respiratory syncytial virus-infected cells, ribavirin inhibited plaque formation at lower concentration when added early postinfection, while higher concentrations were required when added later [16]. Based upon these findings, we speculate that the efficacy of ribavirin treatment in SFTS in vivo may be distinct according to the time point of treatment. Thus, we agree with Shimojima et al. [10] that ribavirin may be used as a prophylactic agent against SFTS, but possibly have limited role for critically-ill SFTS patients. However, further in vivo studies are needed to assess this issue.

In contrast to our in vitro results, a study by Liu et al. [4] reported that ribavirin treatment was not effective in reducing the case-fatality ratio for SFTS patients. However, SFTSV titers were lower in patients who received ribavirin than in those who did not. We suppose that the lack of clinical benefit despite the ribavirin-associated reduction in SFTSV titer may have been due to (1) the therapeutic window: the delay in starting ribavirin treatment after the onset of illness might have led to lack of treatment response as extrapolated based on in vitro results showing ineffective antiviral activity on fully-infected cells [10]; (2) the suboptimal dose of ribavirin (500 mg daily), which was considerably lower than the dose administered to patients with other viral hemorrhagic fevers (2,000 to $4,000 \mathrm{mg}$ daily) [5,6]; or (3) the complex pathogenesis of SFTS, including cytokine storms [17].

It remains to be determined whether the plasma concentrations of ribavirin enough to suppress SFTSV replication can be achieved within the range of permissive dosage. We speculate that it would be possible to reach the inhibitory concentrations for SFTSV replication, if ribavirin is intravenously administered at recommended dosage for other viral hemorrhagic fevers. Single intravenous doses of ribavirin in the range of 600 to $2,400 \mathrm{mg}$ were shown to result in peak serum concentrations of 47 to $161 \mu \mathrm{M}$ (11.5 to $39.3 \mu \mathrm{g} / \mathrm{mL}$ ) within 30 minutes, whereas the same doses of oral ribavirin resulted in much lower peak serum concentrations of 5 to $12 \mu \mathrm{M}$ (1.2 to $2.9 \mu \mathrm{g} /$ $\mathrm{mL}$ ) within 90 minutes [18]. Similarly, in patients with Lassa fever, the mean peak serum levels of ribavirin ranged from 32.1 to $94 \mu \mathrm{M}$ (7.8 to $23.0 \mu \mathrm{g} / \mathrm{mL}$ ) after 4 days of a daily intravenous administration of $4,000 \mathrm{mg}$ and 68 $\mu \mathrm{M}(16.6 \mu \mathrm{g} / \mathrm{mL})$ after 6 days of continued intravenous therapy of $1,500 \mathrm{mg}$ daily [19]. However, the mean serum levels were only 1.2 to $9.6 \mu \mathrm{M}$ (0.3 to $2.3 \mu \mathrm{g} / \mathrm{mL}$ ) in Lassa fever patients who had taken 1,000 $\mathrm{mg}$ of oral ribavirin daily for 10 days [19]. We presume that oral ribavirin treatment is not applicable to the treatment of SFTS, as it takes 2 to 4 weeks to get the steady state and the lower serum concentrations compared with intravenous ribavirin treatment $[6,18]$.

There are a few limitations in our study. First, we used a single cell line in our experiments that we could not compare the range of inhibitory concentrations of ribavirin on viral replication among various cell lines. Although monocytes are known to be the primary target of viral hemorrhagic fever viruses, the preferred target cells of SFTSV have yet to be determined. A previous study demonstrated pathologic changes in the spleen, liver, and kidney of SFTSV-infected C57/BL6 mice and suggested that splenic macrophages were preferentially targeted by the virus [20]. However, a recent study found viral antigen in multiple organs, with the most heavi- 
ly infected tissues being mesenteric lymph nodes and spleen. It also demonstrated that SFTSV did not infect dendritic cells or monocyte/macrophages, but instead primarily infected reticular cells in the spleen [21]. Second, we determined the antiviral efficacy of ribavirin by measuring virus titers in culture supernatant rather than the amount of cell-associated or intracellular SFTSV.

Despite these limitations, our study clearly showed that ribavirin exhibits inhibitory activity against SFTSV replication in vitro. As we have mentioned above, there are issues to be solved before clinical use of ribavirin in SFTS, including the time limit of effective treatment after viral infection and the optimal dosage and route of administration. Since we used in vitro model, antiviral activity and toxicity of ribavirin use in patients with SFTS could not be assured yet. Therefore, further studies using ex vivo and in vivo animal models are warranted to evaluate the potential use of ribavirin for the treatment of SFTS.

\section{KEY MESSAGE}

1. Ribavirin showed a dose-dependent inhibitory effect on severe fever with thrombocytopenia syndrome virus (SFTSV) replication in Vero cells with a half-maximal inhibitory concentration of 3.69 to $8.72 \mu \mathrm{g} / \mathrm{mL}$.

2. The cytopathic effects in SFTSV-infected cells were reduced by ribavirin treatment.

\section{Conflict of interest}

No potential conflict of interest relevant to this article was reported.

\section{REFERENCES}

1. Yu XJ, Liang MF, Zhang SY, et al. Fever with thrombocytopenia associated with a novel bunyavirus in China. $\mathrm{N}$ Engl J Med 2011;364:1523-1532.

2. Takahashi T, Maeda K, Suzuki T, et al. The first identification and retrospective study of severe fever with thrombocytopenia syndrome in Japan. J Infect Dis 2014;209:816827.
3. Kim KH, Yi J, Kim G, et al. Severe fever with thrombocytopenia syndrome, South Korea, 2012. Emerg Infect Dis 2013;19:1892-1894.

4. Liu W, Lu QB, Cui N, et al. Case-fatality ratio and effectiveness of ribavirin therapy among hospitalized patients in china who had severe fever with thrombocytopenia syndrome. Clin Infect Dis 2013;57:1292-1299.

5. Huggins JW. Prospects for treatment of viral hemorrhagic fevers with ribavirin, a broad-spectrum antiviral drug. Rev Infect Dis 1989;11 Suppl 4:S750-S761.

6. Rusnak JM. Experience with ribavirin for treatment and postexposure prophylaxis of hemorrhagic fever viruses: Crimean Congo hemorrhagic fever, lassa fever, and hantaviruses. Appl Biosaf 2011;16:67-87.

7. Oh WS, Heo ST, Kim SH, Choi WJ, Han MG, Kim JY. Plasma exchange and ribavirin for rapidly progressive severe fever with thrombocytopenia syndrome. Int J Infect Dis 2014;18:84-86.

8. Reed LJ, Muench H. A simple method of estimating fifty percent endpoints. Am J Epidemiol 1938;27:493-497.

9. Sun Y, Liang M, Qu J, et al. Early diagnosis of novel SFTS bunyavirus infection by quantitative real-time RT-PCR assay. J Clin Virol 2012;53:48-53.

10. Shimojima M, Fukushi S, Tani H, et al. Effects of ribavirin on severe fever with thrombocytopenia syndrome virus in vitro. Jpn J Infect Dis 2014;67:423-427.

11. Shah NR, Sunderland A, Grdzelishvili VZ. Cell type mediated resistance of vesicular stomatitis virus and Sendai virus to ribavirin. PLoS One 2010;5:e11265.

12. Paragas J, Whitehouse CA, Endy TP, Bray M. A simple assay for determining antiviral activity against Crimean-Congo hemorrhagic fever virus. Antiviral Res 2004;62:21-25.

13. Ozbey SB, Kader C, Erbay A, Ergonul O. Early use of ribavirin is beneficial in Crimean-Congo hemorrhagic fever. Vector Borne Zoonotic Dis 2014;14:300-302.

14. Tasdelen Fisgin N, Ergonul O, Doganci L, Tulek N. The role of ribavirin in the therapy of Crimean-Congo hemorrhagic fever: early use is promising. Eur J Clin Microbiol Infect Dis 2009;28:929-933.

15. Toltzis P, Huang AS. Effect of ribavirin on macromolecular synthesis in vesicular stomatitis virus-infected cells. Antimicrob Agents Chemother 1986;29:1010-1016.

16. Hruska JF, Bernstein JM, Douglas RG Jr, Hall CB. Effects of ribavirin on respiratory syncytial virus in vitro. Antimicrob Agents Chemother 1980;17:770-775.

17. Sun Y, Jin C, Zhan F, et al. Host cytokine storm is associ- 
ated with disease severity of severe fever with thrombocytopenia syndrome. J Infect Dis 2012;206:1085-1094.

18. Fernandez H, Banks G, Smith R. Ribavirin: a clinical overview. Eur J Epidemiol 1986;2:1-14.

19. Bausch DG, Hadi CM, Khan SH, Lertora JJ. Review of the literature and proposed guidelines for the use of oral ribavirin as postexposure prophylaxis for Lassa fever. Clin Infect Dis 2010;51:1435-1441.

20. Jin C, Liang M, Ning J, et al. Pathogenesis of emerging severe fever with thrombocytopenia syndrome virus in C57/BL6 mouse model. Proc Natl Acad Sci U S A 2012;109:10053-10058.

21. Liu Y, Wu B, Paessler S, Walker DH, Tesh RB, Yu XJ. The pathogenesis of severe fever with thrombocytopenia syndrome virus infection in alpha/beta interferon knockout mice: insights into the pathologic mechanisms of a new viral hemorrhagic fever. J Virol 2014;88:1781-1786. 